\title{
Cerebral small vessel disease, cardiovascular risk factors, and future walking speed in old age: a population-based cohort study
}

Emerald G. Heiland ${ }^{1,2,3^{*}}$, Anna-Karin Welmer $2,4,5,6$, Grégoria Kalpouzos ${ }^{2}$, Anna Laveskog ${ }^{7,8}$, Rui Wang ${ }^{2,3}$ and Chengxuan Qiu²*

\begin{abstract}
Background: The purpose of this study was to examine the associations between combined and individual cerebral small vessel disease (CSVD) markers on future walking speed over 9 years; and to explore whether these associations varied by the presence of cardiovascular risk factors (CRFs).

Methods: This population-based cohort study included 331 adults, aged $\geq 60$ years, without limitation in walking speed $(\geq 0.8 \mathrm{~m} / \mathrm{s})$. At baseline, CSVD markers, including white matter hyperintensities (WMH), lacunes, and perivascular spaces (PVS), were assessed on magnetic resonance imaging. The modifiable CRFs (physical inactivity, heavy alcohol consumption, smoking, hypertension, high total cholesterol, diabetes, and overweight/obese) were combined into a score. The association between baseline cSVD markers and the decline in walking speed was examined using linear mixed-effects models, whereas Cox proportional hazards models were used to estimate the association with walking speed limitation (defined as $<0.8 \mathrm{~m} / \mathrm{s}$ ) over the follow-up.
\end{abstract}

Results: Over the follow-up period, 76 (23.0\%) persons developed walking speed limitation. Participants in the highest tertile of the combined cSVD marker score had a hazard ratio (HR) of 3.78 (95\% confidence interval [CI] 1.70-8.45) for walking speed limitation compared with people in the lowest score tertile, even after adjusting for socio-demographics, CRFs, cognitive function, and chronic conditions. When investigating the CSVD markers individually, having the highest burden of WMH was associated with a significantly faster decline in walking speed ( $\beta$ coefficient -0.020 ; 95\% $\mathrm{Cl}-0.035-0.004)$ and a greater HR of walking speed limitation (HR 2.78; 95\% Cl 1.31-5.89) compared with having the lowest WMH burden. Similar results were obtained for the highest tertile of PVS (HR 2.13; 95\% Cl 1.04-4.36). Lacunes were associated with walking speed limitation, but only in men. Having $\geq 4$ CRFs and high WMH volume simultaneously, showed a greater risk of walking speed limitation compared with having $\geq 4$ CRFs and low WMH burden. CRFs did not modify the associations between lacunes or PVS and walking speed.

Conclusions: Combined CSVD markers strongly predict walking speed limitation in healthy older adults, independent of cognitive function, with WMH and PVS being the strongest contributors. Improving cardiovascular health may help to mitigate the negative effects of WMH on future walking speed.

\footnotetext{
*Correspondence: emerald.heiland@surgsci.uu.se; chengxuan.qiu@ki.se

${ }^{1}$ Department of Surgical Sciences, Medical Epidemiology, Uppsala

University, Dag Hammarskjölds väg 14B, 75185 Uppsala, Sweden

${ }^{2}$ Aging Research Center, Department of Neurobiology, Care Sciences

and Society, Karolinska Institutet-Stockholm University, Widerströmska

Huset, Tomtebodavägen 18A, 17165 Solna, Sweden

Full list of author information is available at the end of the article
}

(C) The Author(s) 2021. Open Access This article is licensed under a Creative Commons Attribution 4.0 International License, which permits use, sharing, adaptation, distribution and reproduction in any medium or format, as long as you give appropriate credit to the original author(s) and the source, provide a link to the Creative Commons licence, and indicate if changes were made. The images or other third party material in this article are included in the article's Creative Commons licence, unless indicated otherwise in a credit line to the material. If material is not included in the article's Creative Commons licence and your intended use is not permitted by statutory regulation or exceeds the permitted use, you will need to obtain permission directly from the copyright holder. To view a copy of this licence, visit http://creativecommons.org/licenses/by/4.0/. The Creative Commons Public Domain Dedication waiver (http://creativeco mmons.org/publicdomain/zero/1.0/) applies to the data made available in this article, unless otherwise stated in a credit line to the data. 
Keywords: Cerebral small vessel disease, Walking speed, Physical function, Cardiovascular risk factors, Magnetic resonance imaging $(\mathrm{MRI})$, Population-based cohort study

\section{Introduction}

Walking speed is a fundamental physical function for performing everyday activities of daily living, whereas impairment in this function is a strong predictor of dementia, disability, institutionalisation, and death [13]. Cerebral microvascular lesions are characterised on magnetic resonance imaging (MRI) as markers of cerebral small vessel disease (cSVD) $[4,5]$. These lesions are increasingly common as people age, and are indicative of subclinical abnormalities [6]. Evidence exists that a high burden of cSVD markers is associated with subsequent cognitive impairment among healthy older adults, and can accelerate cognitive decline [7]. Of the cSVD markers, increasing volume of white matter hyperintensities (WMH) has been repeatedly shown to be associated with cognitive impairment and dementia [8]. Moreover, WMH have also been linked with impairment in gait parameters [9-12]. A previous study has shown that clinical markers, such as poor walking speed together with MRI markers of cSVD can predict 8-year mortality [13]. However, WMH may only explain part of the decline in walking speed, as other cSVD markers, which develop concomitantly, may also be associated walking speed impairment and its progressive decline, as demonstrated similarly for cognitive deterioration [7]. In addition, various CSVD markers may represent different stages of pathology although of similar vascular origin.

Apart from WMH, other cSVD markers, such as lacunes and perivascular spaces (PVS) have not been previously investigated in relation to future walking speed, and their combined effect with WMH on walking speed impairment has only been previously examined crosssectionally $[9,12,14]$. Understanding of the association between these imaging markers and prognosis of walking speed can be beneficial clinically and for public health in order to identify older persons at greater risk of poor functional outcomes, so that interventions can be implemented early. This can help older adults to remain independent longer and maintain a good quality of life.

Moreover, age, sex, and cardiovascular risk factors (CRFs) may modify the relationship between the cSVD markers and incipient walking speed impairment $[2,15]$. As cSVD development is correlated with an underlying vascular pathology, a favourable lifestyle may reduce the risk of cSVD progression. In fact, previous findings have revealed that individual and combined CRFs are associated with a greater risk of developing walking speed impairment in older adults [2].
Thus, in this population-based cohort study of older adults, the primary aim was to examine the associations of single cSVD markers and their collective burden with walking speed decline and the development of walking speed limitation over the follow-up period. Additionally, whether these associations varied by age, sex, and combined CRFs were further explored.

\section{Methods}

\section{Study population}

Participants were derived from the population-based Swedish National study on Aging and Care in Kungsholmen (SNAC-K), including adults 60 years and older living in their homes or institutions, in a central area of Stockholm, Sweden. Details on study design and data collection can be found elsewhere, www.snac.org [16]. In the SNAC-K study, at baseline (2001-2004), a random sample of eleven age strata was selected, with 6 years between the younger $(60,66$, and 72 years old) and 3 years between the older age cohorts $(78,81,84,87,90$, and 93 years and older). Follow-up data were collected every 6 years for the younger and every 3 years for the older age cohorts, until 2013 (one examination for the younger cohorts and two examinations for the older cohorts). Data collection was done in this manner due to more rapid changes in health occurring in the older age strata and higher attrition rates. Of the 4590 persons who were alive and eligible, $3363(73.3 \%)$ participated at baseline. This study is based on the brain MRI sub-study embedded in SNACK. Participants in this sub-study were recruited at baseline (September 2001 to October 2003), including 555 persons not living in institutions, and who were not disabled nor diagnosed with dementia (Fig. 1). Compared with the rest of the SNAC-K sample, those who underwent MRI scans, were significantly younger, more men, higher educated, more physically active, never smokers, non-alcohol drinkers, and with a higher body mass index (BMI). However, there was no significant difference found in regards to presence of cardiovascular risk factors (hypertension, diabetes, and dyslipidaemia) [17].

For this study, those with poor image quality $(n=44)$, missing information on at least one cSVD marker $(n=61)$, with baseline walking speed limitation $(<0.8 \mathrm{~m} / \mathrm{s})$ or with missing walking speed information at baseline $(n=46)$, and who were alive and eligible but did not contribute to the follow-up assessments of physical function $(n=73)$, were excluded, leaving an analytical sample of 331 persons. Those who were excluded due to 


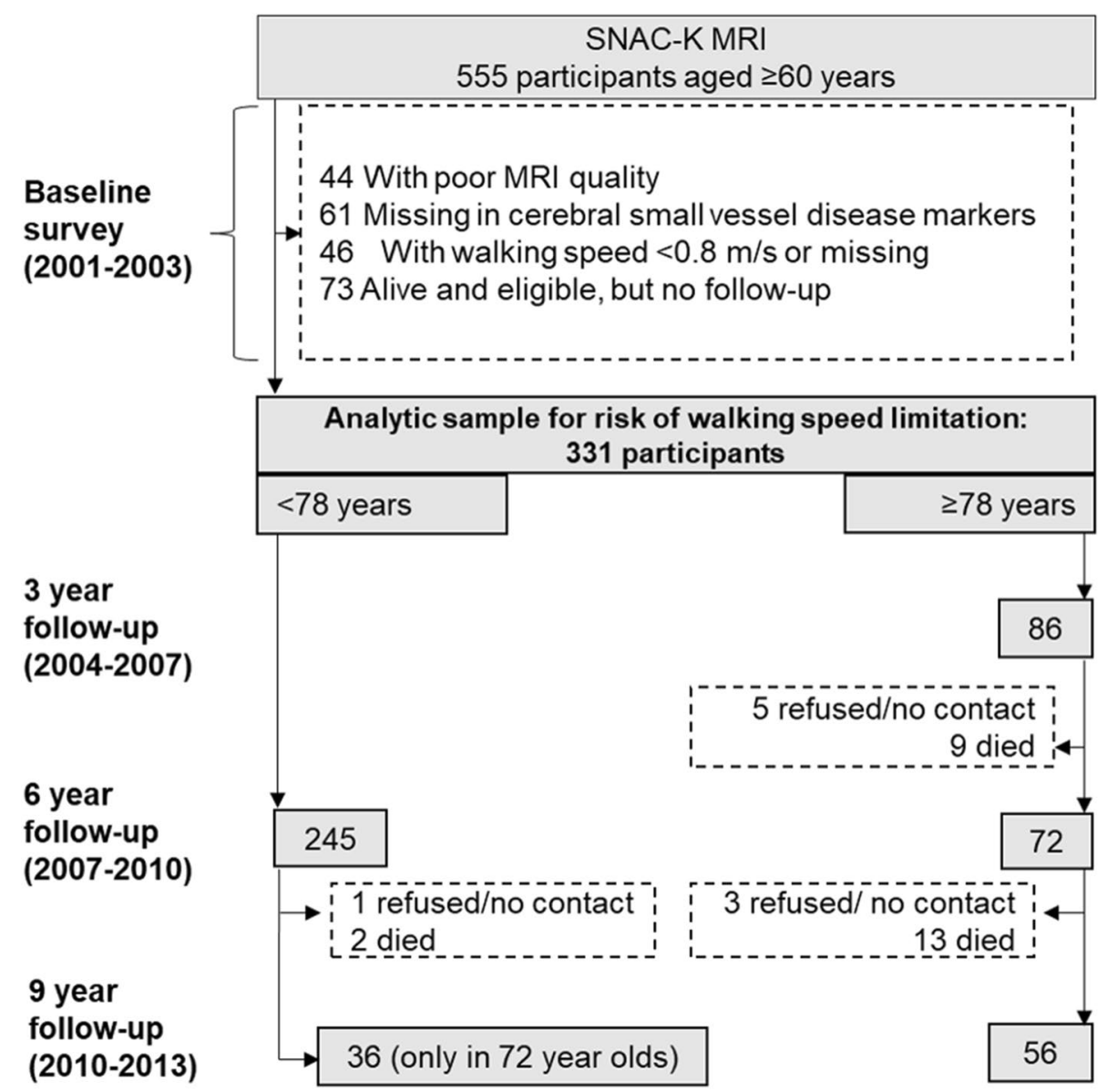

Fig. 1 Flowchart of study population (2001-2004 to 2010-2013). Data were collected every six years for the younger-old (60, 66, and 72years), and every three years for the older-old ( $\geq 78$ years). The older-old had three follow-up examinations, whereas in the younger-old cohort those 72 years old had two follow-up examinations at six and nine years, and those 60 and 66 years old had only one follow-up examination at six years

missing data for the current study were older, had a lower level of education, and a higher number of chronic diseases than those included in the analytical sample.

\section{Ethical approval}

The SNAC-K study was performed in accordance with the Declaration of Helsinki and was approved by the Regional Ethics Review Board in Stockholm. Written and verbal informed consent was provided by all participants.

\section{MRI acquisition and measurements MRI protocol}

Participants were scanned on an Intera $1.5 \mathrm{~T}$ scanner (Philips Healthcare, Best, the Netherlands). The MRI protocol included three sequences: axial three-dimensional T1-weighted, proton-density/T2-weighted, and axial turbo fluid attenuated inversion recovery (FLAIR). The T1-weighted MRI images were acquired with a 3D fast field echo with the following parameters: repetition time [TR] $15 \mathrm{~ms}$, echo time [TE] $7 \mathrm{~ms}$, flip angle [FA] $15^{\circ}$, field of view [FOV] 240, 128 slices with slice thickness $1.5 \mathrm{~mm}$, and in-plane resolution $0.94 \times 0.94 \mathrm{~mm}$, no gap, and matrix $256 \times 256$. The parameters for the a proton-density/T2-weighted sequence were TR $3995 \mathrm{~ms}$, TE $18 / 90 \mathrm{~ms}$, echo-train length 6 , FA $90^{\circ}$, and $603-\mathrm{mm}$ sequential images without a gap or angulation. The parameters for FLAIR were TR $6000 \mathrm{~ms}$, TE $100 \mathrm{~ms}$, inversion time $1900 \mathrm{~ms}$, FA $90^{\circ}$, echo train length 21, FOV 230, 22 slices with slice thickness $5 \mathrm{~mm}$, inplane resolution $0.90 \times 0.90 \mathrm{~mm}$, gap $1 \mathrm{~mm}$, and matrix $256 \times 256$ [17-19].

\section{MRI markers of cerebral small vessel disease}

Three cSVD markers were assessed: WMH, lacunes, and PVS.

1) Global WMH were manually drawn on the FLAIR images by a single rater neuroimaging specialist (G.K.), and further the total volume was calculated after 
interpolation on the corresponding T1-weighted images to compensate for the gap between slices in FLAIR using MRIcron software. Intra-rater reliability was assessed in a subset of 30 individual data sets 2 years later (randomly chosen from SNAC-K MRI sample), which resulted in a correlation of $0.998(P<0.001)$. The Dice coefficient used to determine spatial overlap between two binary images was equal to 0.76 (SD 0.09) for the 30 images, as previously reported [20], which was considered very good agreement between segmentations. T1 images were segmented into grey matter, white matter, and cerebrospinal fluid using SPM12 in MATLAB R2012b. All segments were visually inspected by a neuroimaging expert. WMH volume was adjusted for total brain tissue volume.

2) Lacunes were defined as small lesions with cerebrospinal fluid signal on all sequences and surrounding high signal on FLAIR sequence.

3) PVS were assessed using a visual rating scale, as described in detail earlier [19], where also a description on how to differentiate between lacunes and PVS was explained. Briefly, T1 and T2 images were used to assess PVS in different brain areas (i.e. frontal lobe, parieto-occipital lobe, basal ganglia, including thalami, and subinsular region) in each hemisphere. The number of PVS was scored in each region from 0 to 3: 0 (no visible PVS), 1 (1-5 PVS), 2 (6-10 PVS), or 3 (>10 PVS), with an excellent intra- and inter-rater reliability (both weighted $\kappa=0.77$ ). Scores were then summed to a maximum score of 24 [19]. All lacunes and PVS were assessed by a clinical neuroradiologist.

Cerebral SVD burden was estimated using a similar composite measurement that has been previously reported [7], including WMH, PVS, and lacunes. We assigned 1 point to those who were categorised in the top tertile of WMH volume, in the top tertile of the PVS score, or had lacunes. The scores for each of the three cSVD markers that were concurrently present in a participant were then summed to represent the overall cSVD burden, with the score ranging from 0 to 3.

\section{Assessment of walking speed}

Participants were invited to visit the research centre or data collectors visited the participants in their homes when unable to visit the centre. Walking speed (metres per second, $\mathrm{m} / \mathrm{s}$ ) was tested with a nurse present, who asked the participant to walk at a usual pace over 6 or $2.44 \mathrm{~m}$ [21], using a stop watch and a $2 \mathrm{~m}$ flying start. The shorter distance was used if the participant self-assessed to have a slow usual pace or if the test occurred in their homes where space was restricted. The walking speed test measured with the different distances has been previously reported to be comparable [21], therefore can be used in this manner. Walking speed was examined continuously and dichotomously, with limitation defined according to the well-established cut-off of $<0.8 \mathrm{~m} / \mathrm{s}$ as previously reported $[1,22,23]$.

\section{Covariates}

At baseline, data on potential confounders were collected through interviews, clinical examinations, laboratory tests, and other examinations, by trained staff (physicians, nurses, and psychologists), and retrieved from the Swedish National Patient Register. Demographic data included age, sex, and education (elementary, high school, and university). The modifiable CRFs included physical inactivity, heavy alcohol consumption, ever smoking, hypertension, high total cholesterol, diabetes, and high BMI. Physical inactivity's assessment and categorisation can be found in detail elsewhere [24]. Briefly, physical activity was assessed through a self-administered questionnaire on frequency and intensity in the last 12 months, which was subsequently categorised as inactive, light, and moderate-to-vigorous intensity. Participants were then divided into active (weekly participation in light or moderate-to-vigorous intensity physical activity) and inactive (never or less than 2-3 times/month) groups [24-27]. The subjective measure of physical activity in the SNAC-K study has been shown to have a moderate level of reliability to accelerometry [24]. Alcohol consumption was categorised into no/occasional/light-to-moderate vs. heavy ( $>14$ drinks per week for men or $>7$ drinks per week for women) [28]. Smoking status was recorded as never or ever. Seated arterial blood pressure was measured twice on the left arm with a sphygmomanometer, and the mean of two measurements was used for the analysis. Systolic blood pressure (SBP) and diastolic blood pressure (DBP) were categorised according to a previous study [29] (SBP: <130, 130-139, 140-149, and $\geq 150 \mathrm{mmHg}$; DBP: $<70,70-79,80-89$, and $\geq 90 \mathrm{mmHg}$ ). All medications were classified according to the Anatomical Therapeutic Chemical (ATC) classification system. Hypertension was defined as $\geq 140 / 90 \mathrm{mmHg}$ or use of antihypertensive agents (ATC codes $\mathrm{C} 02, \mathrm{C} 03$, and $\mathrm{C07}-\mathrm{C} 09$ ). BMI was categorised as underweight $\left(<20 \mathrm{~kg} / \mathrm{m}^{2}\right)$, normal (2024.9), overweight (25-29.9), or obese ( $\geq 30)$. High total cholesterol was defined as a level $\geq 6.22 \mathrm{mmol} / \mathrm{l}$ or use of cholesterol lowering agents (ATC code C10). Diabetes was defined according to self-reported history, records from the Swedish National Patient Register, the use of hypoglycaemic agents (ATC code A10), or a glycated haemoglobin level $\geq 6.5 \%$ [30].

In addition, C-reactive protein, a marker of inflammation in the body, was measured following a standard protocol, with a high concentration defined as greater than $5 \mathrm{mg} / \mathrm{L}$ [31]. Global cognitive function was assessed using the Mini-Mental State Examination (MMSE) 
administered by a psychologist [32]. Number of chronic diseases (e.g. cancer and osteoporosis) and the presence of cardio- and cerebrovascular diseases (CVDs; i.e. coronary heart disease, atrial fibrillation, heart failure, and cerebrovascular disease) at baseline were defined by integrating information from clinical examination, electrocardiogram, medication use, and the Swedish National Patient Register [33].

\section{Statistical analysis}

Cross-sectional associations between baseline walking speed and the combined and individual cSVD markers were assessed using the general linear model. In order to investigate the average annual change in walking speed $(\mathrm{m} / \mathrm{s})$ associated with baseline cSVD markers, linear mixed-effects models were employed using an interaction term between the cSVD markers and followup time. Model 1 was unadjusted, whereas model 2 was adjusted for age, sex, education, CRFs, MMSE, number of chronic diseases, CRP, and CVDs. Cox proportional hazards models were performed to investigate the association between individual cSVD markers and their combined burden and incident walking speed limitation, using follow-up time as the time scale. Proportionality was satisfied for all models. Events were censored at first occurrence, otherwise censoring occurred at the end of the follow-up, or when death or dropout occurred. A dummy variable was created for variables with missing information of a covariate $(7.2 \%$ missing), in order to maximise the sample size. We tested interactions of cSVD markers with age and sex on walking speed limitation in additional Cox models. If the interaction was significant $(P \leq 0.05)$, a subsequent stratified analysis was performed. A CRF score was also calculated by counting the number of the dichotomised CRFs (i.e. hypertension, diabetes, heavy alcohol consumption, physical inactivity, smoking, and high cholesterol). Then, the CRF score was dichotomised based on the median $(<4$ or $\geq 4$ CRFs $)$ and stratified analyses were performed to test whether the association between each individual cSVD marker on future walking speed limitation and decline varied by combined CRFs, using Cox models and linear mixedeffects models.

Additional sensitivity analyses were performed to determine if death was a competing risk in additional Cox models. Statistical software Stata version 15 (StataCorp, College Station, TX), was used for all analyses. $P$ value for significance was set at $\leq 0.05$.

\section{Results}

At baseline, the mean age of the 331 participants was 68.9 (SD 8.3) years and 58.3\% were women. Compared with those who did not develop walking speed limitation at follow-up, those who did were more likely to be older, have a lower education and MMSE score, have more chronic diseases, were not smokers, had a higher average SBP, taking anti-hyperintensive agents, and were more likely to have cerebrovascular disease and heart failure (Table 1). At baseline, neither individual nor combined cSVD markers were significantly associated with walking speed (data not shown).

Linear mixed-effects modelling analysis showed a faster average annual decline in walking speed for those in the third tertile of WMH volume compared with the first tertile ( $\beta$ coefficient -0.020 ; 95\% confidence interval $[\mathrm{CI}]-0.035,-0.004)$, even after adjusting for sociodemographics, CRFs, CRP, number of chronic diseases, CVDs, and MMSE score (Fig. 2). Lacunes and PVS were not significantly associated with walking speed decline. When the three cSVD markers were aggregated into a composite score, the $\beta$ coefficient (average annual decline) of walking speed was - 0.018 (95\% CI -0.030, $-0.004)$ for having a low cSVD score and -0.021 (95\% CI $-0.040,-0.004$ ) for having a high cSVD score.

During an average of 6.0 (SD 1.4) years of follow-up, 76 persons $(23.0 \%)$ developed walking speed limitation, with the incidence being 3.83 per 100 person-years. Of these, the average age was 77.7 (SD 6.3) years at baseline and $67.1 \%$ were women. The results from the Cox regression analysis showed that after full adjustment, greater WMH volume was significantly associated with a greater risk of developing walking speed limitation (Hazard Ratio [HR] 2.78; 95\% CI 1.31-5.89) (Table 2). Likewise, an increased risk of incident walking speed limitation was shown for those in the highest tertile of the PVS score, compared with those in the lowest tertile (HR 2.13; 95\% CI 1.04-4.36). No statistically significant association was found between lacunes and walking speed limitation. An increasing cSVD score was significantly associated with an increased HR of subsequent walking speed limitation in a dose-dependent manner ( $P$ for trend $=0.001$ ). The highest cSVD score was associated with an HR of walking speed limitation of 3.78 (95\% CI 1.70-8.45).

There were no statistically significant interactions between any of the single cSVD markers and age on incident walking speed limitation. A statistically significant modifying effect of sex $(P=0.032)$ was observed for the association between lacunes and walking speed, such that men with lacunes were more likely to develop walking speed limitation than women in the fully-adjusted models (Men: HR 13.81; 95\% CI 1.62-117.99; Women: HR 1.01; 95\% CI 0.40-2.56). However, there was no difference in walking speed decline between men and women according to the presence of baseline lacunes. The sensitivity analysis, considering death as a competing event, 
Table 1 Baseline characteristics of the total sample and stratification by follow-up walking speed limitation

\begin{tabular}{|c|c|c|c|c|}
\hline \multirow[t]{2}{*}{ Characteristics } & \multirow{2}{*}{$\begin{array}{l}\text { Total sample } \\
(n=331)\end{array}$} & \multicolumn{3}{|c|}{ Follow-up walking speed limitation } \\
\hline & & No $(n=255)$ & Yes $(n=76)$ & $P$ value \\
\hline Age (years), mean (SD) & $68.9(8.3)$ & $66.2(6.8)$ & $77.7(6.3)$ & $<0.001$ \\
\hline Women, n (\%) & $193(58.31)$ & $142(55.7)$ & $51(67.1)$ & 0.08 \\
\hline Education, n (\%) & & & & 0.01 \\
\hline Elementary & $32(9.7)$ & $22(8.6)$ & $10(13.2)$ & \\
\hline High School & $141(42.6)$ & $100(39.2)$ & $41(54.0)$ & \\
\hline University & $158(47.7)$ & $133(52.2)$ & $25(32.9)$ & \\
\hline MMSE score ${ }^{a}$, mean (SD) & $29.3(0.9)$ & $29.3(0.9)$ & $29.0(1.1)$ & 0.02 \\
\hline High CRP ${ }^{a}, n(\%)$ & $54(16.6)$ & $38(15.2)$ & $16(21.1)$ & 0.23 \\
\hline Number of chronic diseases, mean (SD) & $3.2(2.0)$ & $3.0(1.8)$ & $5.0(2.2)$ & $<0.001$ \\
\hline Physically inactive, n (\%) & $54(16.3)$ & $43(16.9)$ & $11(14.5)$ & 0.62 \\
\hline Heavy alcohol consumption, n (\%) & 65 (19.6) & $51(20.0)$ & $14(18.4)$ & 0.76 \\
\hline Ever smoking, n (\%) & $186(56.2)$ & $152(59.6)$ & $34(44.7)$ & 0.02 \\
\hline $\mathrm{SBP}$, mean (SD) & $141.4(19.1)$ & $139.7(18.6)$ & $147.3(19.5)$ & 0.002 \\
\hline DBP, mean (SD) & $83.2(10.0)$ & $83.3(10.0)$ & $82.7(10.0)$ & 0.62 \\
\hline Anti-hypertensive agents, n (\%) & $108(32.6)$ & $68(26.7)$ & $40(52.6)$ & $<0.001$ \\
\hline Diabetes, n (\%) & $22(6.7)$ & $16(6.3)$ & $6(7.9)$ & 0.62 \\
\hline High total cholesterol ${ }^{\mathrm{a}}, \mathrm{n}(\%)$ & $192(58.7)$ & $146(58.2)$ & $46(60.5)$ & 0.71 \\
\hline BMI $\left(k g / m^{2}\right), n(\%)$ & & & & 0.47 \\
\hline Underweight $(<20)$ & $12(3.6)$ & $11(4.3)$ & $1(1.3)$ & \\
\hline Normal (20-24.9) & $134(40.5)$ & 99 (38.8) & $35(46.1)$ & \\
\hline Overweight (25-29.9) & $147(44.4)$ & $116(45.5)$ & $31(40.8)$ & \\
\hline Obese $(\geq 30)$ & $38(11.5)$ & $29(11.4)$ & $9(11.8)$ & \\
\hline Atrial fibrillation, n (\%) & $17(5.1)$ & $13(5.1)$ & $4(5.3)$ & 0.95 \\
\hline Coronary heart disease, n (\%) & $24(7.3)$ & $19(7.5)$ & $5(6.6)$ & 0.80 \\
\hline Heart failure, n (\%) & $13(3.9)$ & $6(2.4)$ & $7(9.2)$ & 0.01 \\
\hline Cerebrovascular diseases, n (\%) & $10(3.0)$ & $5(2.0)$ & $5(6.6)$ & 0.04 \\
\hline WMH volume ${ }^{b}$, mean (SD) & $0.8(1.3)$ & $0.6(1.2)$ & $1.3(1.3)$ & $<0.001$ \\
\hline WMH volume, n (\%) & & & & 0.001 \\
\hline 1st Tertile & $118(35.7)$ & $101(39.6)$ & $17(22.4)$ & \\
\hline 2nd Tertile & $126(38.1)$ & $99(38.8)$ & $27(35.5)$ & \\
\hline 3rd Tertile & $87(26.3)$ & $55(21.6)$ & $32(42.1)$ & \\
\hline PVS score, mean (SD) & $15.6(4.5)$ & $15.4(4.6)$ & $16.2(4.5)$ & 0.20 \\
\hline PVS, n (\%) & & & & 0.46 \\
\hline 1st Tertile & $103(31.1)$ & $82(32.2)$ & $21(27.6)$ & \\
\hline 2nd Tertile & $121(36.6)$ & $95(37.3)$ & $26(34.2)$ & \\
\hline 3rd Tertile & $107(32.3)$ & $78(30.6)$ & $29(38.2)$ & \\
\hline Presence of lacunes, n (\%) & $37(11.2)$ & $22(8.6)$ & $15(19.7)$ & 0.01 \\
\hline cSVD burden, n (\%) & & & & $<0.001$ \\
\hline 0 & $159(48.0)$ & $138(54.1)$ & $21(27.6)$ & \\
\hline 1 & $118(35.7)$ & $82(32.2)$ & $36(47.4)$ & \\
\hline 2 or 3 & $54(16.3)$ & $35(13.7)$ & $19(25.0)$ & \\
\hline
\end{tabular}

${ }^{a}$ Data were missing for 15 persons in MMSE score, 5 in CRP, and 4 in total cholesterol

b Volume was corrected for total brain tissue volume

SD standard deviation, MMSE Mini-Mental State Examination, WMH white matter hyperintensities, $P V S$ perivascular spaces, $C R P C$-reactive protein, SBP systolic blood pressure, $D B P$ diastolic blood pressure, $B M I$ body mass index, $C S V D$ cerebral small vessel disease

$P$ value is for the test of comparisons between people with and without incident walking speed limitation during the follow-up period 


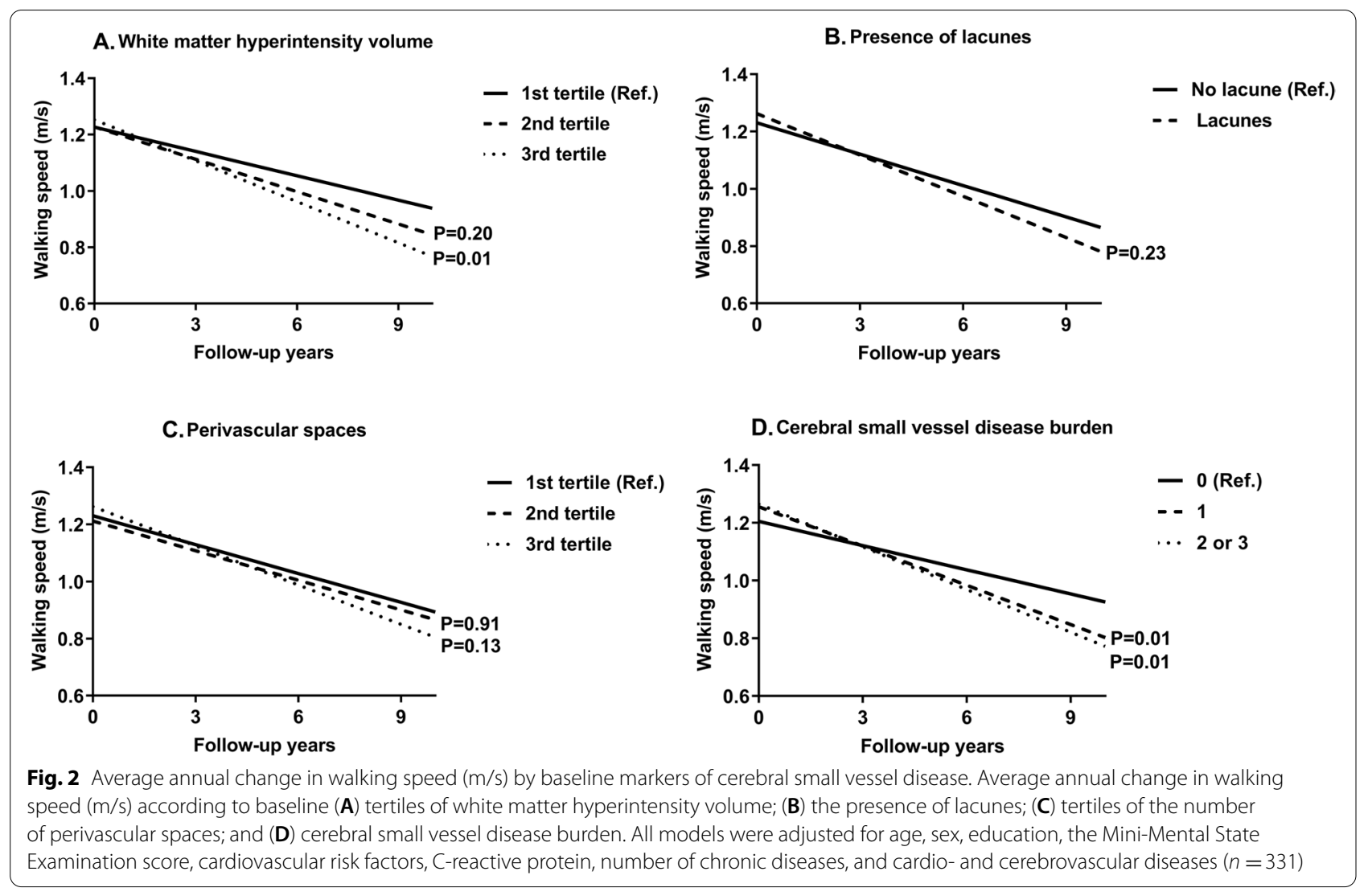

Table 2 Individual and combined cerebral small vessel disease markers and incident walking speed limitation $(n=331)$

\begin{tabular}{|c|c|c|c|c|c|}
\hline \multirow[t]{2}{*}{ cSVD markers } & & \multirow{2}{*}{$\begin{array}{l}\text { No. of } \\
\text { subjects }\end{array}$} & \multirow{2}{*}{$\begin{array}{l}\text { No. of } \\
\text { cases }\end{array}$} & \multicolumn{2}{|c|}{ Hazard Ratio (95\% Confidence Interval) } \\
\hline & & & & Model $1^{\mathrm{a}}$ & Model $2^{\mathrm{a}}$ \\
\hline WMH volume, continuous & & 331 & 76 & $1.38(1.15-1.66)$ & $1.40(1.11-1.76)$ \\
\hline \multirow[t]{4}{*}{ WMH volume } & 1st Tertile & 118 & 17 & 1.00 (Ref.) & 1.00 (Ref.) \\
\hline & 2nd Tertile & 126 & 27 & $1.31(0.71-2.41)$ & $1.70(0.77-3.79)$ \\
\hline & 3rd Tertile & 87 & 32 & $2.35(1.30-4.25)$ & $2.78(1.31-5.89)$ \\
\hline & $P$ for trend & & & 0.003 & 0.01 \\
\hline \multirow[t]{2}{*}{ Lacunes } & No & 294 & 61 & 1.00 (Ref.) & 1.00 (Ref.) \\
\hline & Yes & 37 & 15 & $1.81(1.02-3.21)$ & $1.43(0.70-2.92)$ \\
\hline PVS score, continuous & & 331 & 76 & $1.02(0.97-1.08)$ & $1.08(1.01-1.15)$ \\
\hline \multirow[t]{4}{*}{ PVS score } & 1st Tertile & 103 & 21 & 1.00 (ref.) & 1.00 (Ref.) \\
\hline & 2nd Tertile & 121 & 26 & $0.81(0.45-1.44)$ & $1.15(0.57-2.32)$ \\
\hline & 3rd Tertile & 107 & 29 & $1.21(0.69-2.13)$ & $2.13(1.04-4.36)$ \\
\hline & $P$ for trend & & & 0.45 & 0.04 \\
\hline cSVD burden, continuous & & 331 & 76 & $1.62(1.21-2.16)$ & $1.95(1.31-2.89)$ \\
\hline \multirow[t]{4}{*}{ CSVD burden } & 0 & 159 & 21 & 1.00 (Ref.) & 1.00 (Ref.) \\
\hline & 1 & 118 & 36 & $2.36(1.38-4.05)$ & $2.34(1.20-4.58)$ \\
\hline & 2 or 3 & 54 & 19 & $2.58(1.38-4.81)$ & $3.78(1.70-8.45)$ \\
\hline & $P$ for trend & & & 0.001 & 0.001 \\
\hline
\end{tabular}

a Model 1: unadjusted; Model 2: adjusted for age, sex, education, the Mini-Mental State Examination score, cardiovascular risk factors (i.e., physical inactivity, heavy alcohol consumption, smoking, hypertension, body mass index, cholesterol, diabetes), C-reactive protein, number of chronic diseases, and cardio- and cerebrovascular diseases

CSVD cerebral small vessel disease, WMH white matter hyperintensities, PVS perivascular spaces 
yielded similar results to the initial analyses (Supplementary Table 1).

The association between WMH volume and walking speed limitation varied by CRF burden, such that those with $\geq 4$ CRFs and were in the highest tertile of WMH volume had a much greater risk for future walking speed limitation (HR 7.87; 95\% CI 1.07-57.97) compared with those in the lowest tertile of WMH volume and with $\geq 4$ CRFs (Fig. 3A). In addition, compared with those with low WMH burden and $\geq 4$ CRFs, those with high WMH burden and $\geq 4$ CRFs indicated a potentially faster decline in walking speed over the follow-up $(P=0.06)$ (Fig. 3B). CRFs did not modify the associations between lacunes or PVS and walking speed.

\section{Discussion}

In this population-based cohort study of older adults without walking speed limitation at baseline, having a greater burden of global cSVD markers simultaneously at baseline was associated with an accelerated decline in walking speed over 9 years of follow-up. Particularly, a greater WMH burden was associated with a faster walking speed decline and future walking speed limitation, independent of demographic factors, cognitive function, chronic conditions, cardio- and cerebrovascular diseases, and other cardiovascular risk factors. A larger number of PVS was also associated with an increased risk of developing walking speed limitation over the follow-up, and lacunes were associated with a higher risk of walking speed limitation only in men. These results not only confirm the association between WMH on the development of poor walking speed, but also demonstrate the potential devastating effects of PVS and lacunes alone and the combined burden of cerebral microvascular abnormalities on age-related changes in walking speed. In addition, having a poor cardiovascular profile together with a high burden of WMH substantially increased the risk of walking speed limitation. Therefore, improvement in modifiable CRFs may help reduce the risk of walking speed limitation associated with WMH.

This is one of the first longitudinal investigations on the association between the burden of multiple cSVD markers and future walking speed among a physically intact older population aged 60 to 90 years. The association between a greater cSVD burden and worse performance on walking speed has been previously reported only in a cross-sectional study [9], where WMH were the main driving cSVD marker. Cerebral SVD burden has also been investigated in association with cognitive decline and dementia in an earlier study from the SNAC-K MRI cohort, revealing that a higher burden of CSVD markers was strongly associated with future cognitive decline and dementia [7]. This may indicate a shared neuropathological basis in the decline of walking speed and cognitive function in older adults.

Population-based cohort studies on the longitudinal associations between the presence of lacunes and walking speed are lacking. Earlier cross-sectional studies have shown inconsistent findings on the association between lacunes and walking speed, with some studies observing an association [12, 34], while others not $[9,35]$. In the present study, a significant association was seen only in
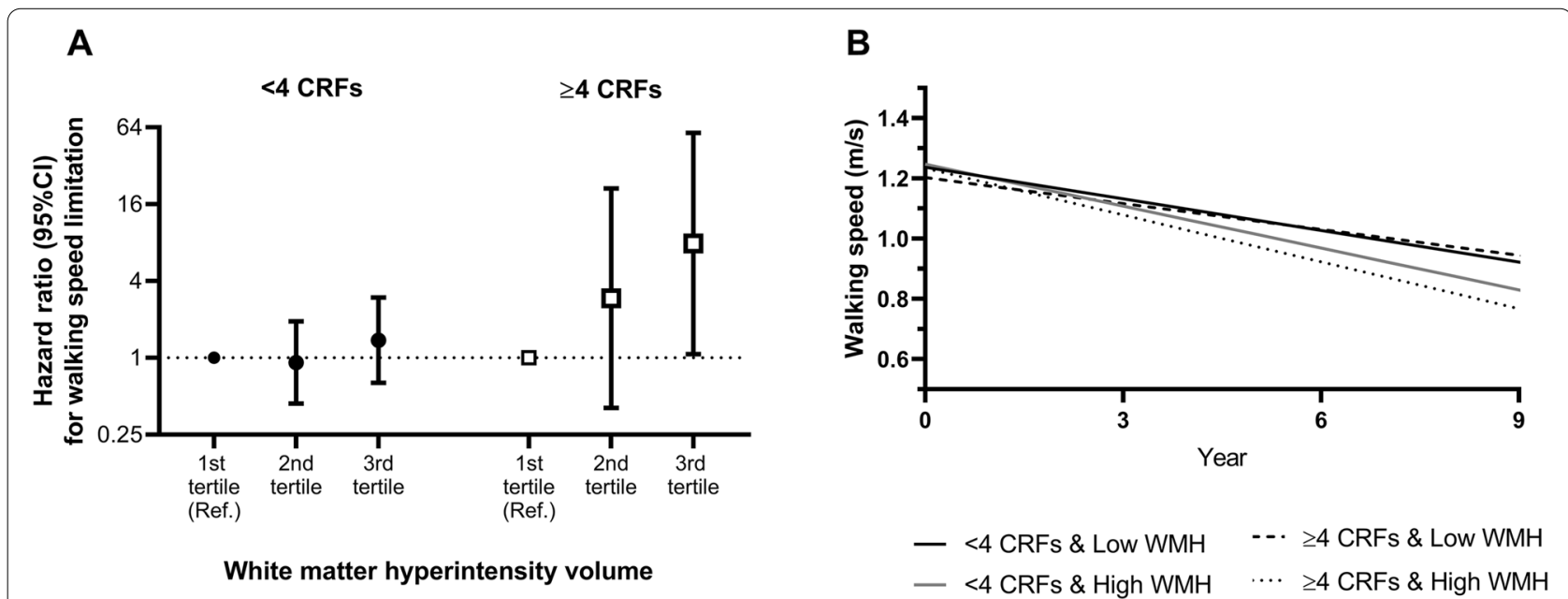

Fig. 3 The association between white matter hyperintensity volume and walking speed limitation and decline, by cardiovascular risk factor burden. Association between white matter hyperintensity volume $(\mathrm{WMH})$ and $(\mathbf{A})$ future walking speed limitation and $(\mathbf{B})$ walking speed decline $(\mathrm{m} / \mathrm{s})$ by cardiovascular risk factor burden. All models were adjusted for age, sex, education, the Mini-Mental State Examination score, C-reactive protein, number of chronic diseases, and cardio- and cerebrovascular diseases. CRFs = cardiovascular risk factors; WMH = white matter hyperintensities; $\mathrm{Cl}=$ confidence interval 
men. Men are more likely to have a poorer vascular profile than women, which may account for a more advanced vascular pathology. The men in the present study were more likely to have diabetes, be obese, and have cardiovascular disease compared with the women (Supplementary Table 2). However, the association between lacunes and walking speed was present after adjusting for CRFs. Thus, other covert pathological pathways may be at play such as cerebral blood flow and glucose metabolism. Furthermore, enlarged PVS are increasingly being recognised as a marker of cSVD and have been associated with cognitive decline in healthy older adults $[19,36]$, but cross-sectional studies have exhibited no correlation between PVS and walking speed $[9,35]$. Thus, a high burden of PVS may be more correlated with cognitive than physical decline, but may indicate risk of incident walking speed limitation, as observed in the present study.

The strong association observed between cSVD markers and walking speed decline supports vascular brain injury to be a neuropathological basis for physical function diminishment in old age. This in turn reflects the robustness of walking speed as a predictor of many adverse health outcomes [1]. Walking speed is a known superior, simple tool of physical function, and therefore, can be considered as a reliable substitute in clinical and general population settings where a full physical function battery of tests is not feasible to implement. Walking speed assesses not only the physical health of older adults, but also underlying pathologies in the absence of overt conditions. Therefore, using a simple and valid walking speed tool, as in the present study, can reduce administrative burden and increase feasibility for use in more ecologically valid settings.

A potential underlying mechanism in which a greater WMH burden may promote the decline in walking speed is through changes in white matter microstructural integrity. Emerging evidence suggests that greater microstructural integrity of normal-appearing white matter may be a key factor in the preservation of physical function [10]. One study of community-dwelling older adults found that those with a high WMH burden and low microstructural white matter integrity had slower walking speeds [10]. Other mechanisms may be pertaining to the "disconnection syndrome". This theory suggests that WMH compromise sensorimotor function by disrupting the complex neural networks in cortical regions with projection and association fibres involved in motor control, especially in the frontal regions [37, 38]. Furthermore, slow walkers have been observed to also have reduced neurovascular coupling. However, older adults with WMH who demonstrate effective alignment of cerebral blood flow with cognitively demanding tasks indicated a better preservation of walking speed [39]. Moreover, it is known that older adults recruit more brain regions when undertaking motor tasks than young people, and that WMH accrual may heighten the decline in neural plasticity and lead to physical impairment [37]. More studies are required to disentangle the specific brain regions involved in the decline of walking speed. Additionally, CRFs are known to be strongly associated with cSVD markers and a high CRF burden may exacerbate the association between WMH and walking speed limitation. Thus, improvements on modifiable CRFs may help in slowing the decline in walking speed due to CSVD. In fact, a multi-domain intervention targeting several CRFs simultaneously found improvements in cognitive performance over 2 years in older adults [40]. In addition, a randomised controlled trial including persons with early Alzheimer's disease found that a lifestyle intervention aimed at improving CRFs, such as BMI, physical inactivity, smoking, and hypertension, slowed the progression of WMH compared with those who received usual care [41]. Thus, these modifiable lifestyle factors may admittedly be potential targets for decreasing the risk of future walking speed impairment among older adults.

Strengths of our study are the prospective design and the population-based cohort of older adults, as well as the objective measure of walking speed. However, our study was not without limitations. The lack of additional cSVD markers such as cerebral microbleeds and microinfarcts may have underestimated the association of a burden of cSVD with physical dysfunction. These additional cSVD markers were not included in the present study due to the lack of respective MRI sequences in the SNAC-K MRI study. Some of the CRFs were self-reported, which could lead to an overestimation, particularly for physical activity. Underestimation may have occurred due to dropouts, owing to their greater likelihood of having more cSVD and poorer physical function. In addition, the data were limited by only having MRI markers and CRFs available at baseline. Also, exclusion of people with walking speed limitation at baseline might affect our results on walking speed. However, the sensitivity analysis including persons with walking speed limitation at baseline yielded similar results to the main analysis (Supplementary Fig. 1). Finally, caution is needed when generalising our findings to other populations due to the high socioeconomic position of our study sample.

\section{Conclusions}

This population-based cohort study showed that a high burden of cSVD markers is associated with accelerated walking speed decline and an increased risk of walking speed limitation among older adults. Additionally, improving CRFs may help in mitigating the negative effects of WMH on walking speed. This provides greater 
understanding into the potential neuropathological pathways underpinning the decline in walking speed in older adults, and thus, paves the way for the development of potential interventions to help older adults maintain functional independence into later life.

\section{Supplementary Information}

The online version contains supplementary material available at https://doi. org/10.1186/s12883-021-02529-6.

Additional file 1: Supplementary Table 1. Competing risk analysis. Supplementary Table 2. Baseline characteristics of the total sample and stratification by sex. Supplementary Fig. 1. Average annual change in walking speed by baseline markers of cerebral small vessel disease including individuals with walking speed limitation at baseline.

\section{Acknowledgements}

We thank the SNAC-K participants and data collectors for their contribution and collaboration.

\section{Authors' contributions}

Study design and concept: EGH, AKW, and CQ; Data analysis and interpretation: EGH and RW; Data acquisition and interpretation: AKW, GK, and AL. Manuscript preparation and drafting: EGH; Manuscript revision for intellectual content: EGH, AKW, GK, AL, RW, and CQ. The author(s) read and approved the final manuscript.

\section{Funding}

Open access funding provided by Uppsala University. SNAC was supported by the Swedish Research Council, the Swedish Ministry of Health and Social Affairs, and the participating county councils and municipalities. This work was also partly supported by the Swedish Research Council for Health, Working Life, and Welfare (project no: 2014-1382), the Swedish Research Council (project no.: 2015-02531 and 2017-05819), Gun and Bertil Stohnes Stiftelse, Stiftelsen Ragnhild and Einar Lundströms Minne, and Karolinska Institutet (KID-funding), Stockholm, Sweden. The funding sources had no involvement in the study design, nor in collection, analysis, or interpretation of the data, or writing of the manuscript. This study was accomplished within the context of the Swedish National Graduate School for Competitive Science on Ageing and Health (SWEAH) funded by the Swedish Research Council, Stockholm, Sweden

\section{Availability of data and materials}

The datasets during the current study are not publicly available, but are available upon reasonable request through the SNAC-K website: https://www. snac-k.se/for-researchers/application-form/.

\section{Declarations}

\section{Ethics approval and consent to participate}

The SNAC-K study was performed in accordance with the Declaration of Helsinki and was approved by the Regional Ethics Review Board in Stockholm. Written and verbal informed consent was provided by all participants.

\section{Consent for publication}

Not applicable.

\section{Competing interests}

None.

\section{Author details}

'Department of Surgical Sciences, Medical Epidemiology, Uppsala University, Dag Hammarskjölds väg 14B, 75185 Uppsala, Sweden. ${ }^{2}$ Aging Research Center, Department of Neurobiology, Care Sciences and Society, Karolinska Institutet-Stockholm University, Widerströmska Huset, Tomtebodavägen 18A, 17165 Solna, Sweden. ${ }^{3}$ Department of Physical Activity and Health,
The Swedish School of Sport and Health Sciences (GIH), Lidingövägen 1, 114 86 Stockholm, Sweden. ${ }^{4}$ Stockholm Gerontology Research Center, Sveavägen 155, 11346 Stockholm, Sweden. ${ }^{5}$ Women's Health and Allied Health Professionals Theme, Medical Unit Medical Psychology, Karolinska University Hospital, Stockholm, Sweden. ${ }^{6}$ Division of Physiotherapy, Department of Neurobiology, Care Sciences and Society, Karolinska Institutet, Alfred Nobels allé 23, 14183 Huddinge, Sweden. ${ }^{7}$ Division of Neuro, Department of Clinical Neuroscience, Karolinska Institutet, Tomtebodavägen 18A, 17165 Solna, Sweden. ${ }^{8}$ Department of Neuroradiology, Karolinska University Hospital, Eugeniavägen 3, 17176 Solna, Sweden.

Received: 5 July 2021 Accepted: 13 December 2021

Published online: 24 December 2021

\section{References}

1. Abellan van Kan G, Rolland Y, Andrieu S, Bauer J, Beauchet O, et al. Gait speed at usual pace as a predictor of adverse outcomes in communitydwelling older people an international academy on nutrition and aging (IANA) task force. J Nutr Health Aging. 2009;13(10):881-9.

2. Heiland EG, Qiu C, Wang R, Santoni G, Liang Y, et al. Cardiovascular risk burden and future risk of walking speed limitation in older adults. J Am Geriatr Soc. 2017:65(11):2418-24.

3. Kueper JK, Speechley M, Lingum NR, Montero-Odasso M. Motor function and incident dementia: a systematic review and meta-analysis. Age Ageing. 2017;46(5):729-38.

4. Pantoni L. Cerebral small vessel disease: from pathogenesis and clinical characteristics to therapeutic challenges. Lancet Neurol. 2010;9(7):689-701.

5. Wardlaw JM, Smith EE, Biessels GJ, Cordonnier C, Fazekas F, et al. Neuroimaging standards for research into small vessel disease and its contribution to ageing and neurodegeneration. Lancet Neurol. 2013;12(8):822-38.

6. de Leeuw FE, de Groot JC, Achten E, Oudkerk M, Ramos LM, et al. Prevalence of cerebral white matter lesions in elderly people: a population based magnetic resonance imaging study. The Rotterdam scan study. J Neurol Neurosurg Psychiatry. 2001;70(1):9-14.

7. Wang R, Laveskog A, Laukka EJ, Kalpouzos G, Backman L, et al. MRI load of cerebral microvascular lesions and neurodegeneration, cognitive decline, and dementia. Neurology. 2018;91(16):e1487-e97.

8. Alber J, Alladi S, Bae H-J, Barton DA, Beckett LA, et al. White matter hyperintensities in vascular contributions to cognitive impairment and dementia (VCID): Knowledge gaps and opportunities. Alzheimer's Dement (New York, NY). 2019:5:107-17.

9. Pinter D, Ritchie SJ, Doubal F, Gattringer T, Morris Z, et al. Impact of small vessel disease in the brain on gait and balance. Sci Rep. 2017;7:41637.

10. Rosario BL, Rosso AL, Aizenstein HJ, Harris T, Newman AB, et al. Cerebral white matter and slow gait: contribution of Hyperintensities and Normalappearing parenchyma. J Gerontol A Biol Sci Med Sci. 2016;71 (7):968-73.

11. Zheng JJ, Delbaere K, Close JC, Sachdev PS, Lord SR. Impact of white matter lesions on physical functioning and fall risk in older people: a systematic review. Stroke. 2011;42(7):2086-90.

12. Smith $E E, O^{\prime}$ Donnell M, Dagenais $G$, Lear SA, Wielgosz A, et al. Early cerebral small vessel disease and brain volume, cognition, and gait. Ann Neurol. 2015;77(2):251-61.

13. van der Holst HM, van Uden IWM, Tuladhar AM, de Laat KF, van Norden AGW, et al. Factors associated with 8-year mortality in older patients with cerebral small vessel disease: the Radboud University Nijmegen diffusion tensor and magnetic resonance cohort (RUN DMC) study. JAMA Neurol. 2016;73(4):402-9

14. Stijntjes M, de Craen AJ, van der Grond J, Meskers CG, Slagboom PE, et al. Cerebral microbleeds and lacunar infarcts are associated with walking speed independent of cognitive performance in middle-aged to older adults. Gerontology. 2016;62(5):500-7.

15. Soumare A, Tavernier B, Alperovitch A, Tzourio C, Elbaz A. A cross-sectional and longitudinal study of the relationship between walking speed and cognitive function in community-dwelling elderly people. J Gerontol A Biol Sci Med Sci. 2009;64(10):1058-65.

16. Lagergren M, Fratiglioni L, Hallberg IR, Berglund J, Elmstahl S, et al. A longitudinal study integrating population, care and social services data. 
The Swedish national study on aging and care (SNAC). Aging Clin Exp Res. 2004;16(2):158-68.

17. Wang R, Fratiglioni L, Kalpouzos G, Lovden M, Laukka EJ, et al. Mixed brain lesions mediate the association between cardiovascular risk burden and cognitive decline in old age: a population-based study. Alzheimers Dement. 2017;13(3):247-56.

18. Zhang Y, Qiu C, Lindberg O, Bronge L, Aspelin P, et al. Acceleration of hippocampal atrophy in a non-demented elderly population: the SNAC-K study. Int Psychogeriatr. 2010;22(1):14-25.

19. Laveskog A, Wang R, Bronge L, Wahlund LO, Qiu C. Perivascular spaces in old age: assessment, distribution, and correlation with white matter Hyperintensities. AJNR Am J Neuroradiol. 2018;39(1):70-6.

20. Kohncke Y, Laukka EJ, Brehmer Y, Kalpouzos G, Li TQ, et al. Three-year changes in leisure activities are associated with concurrent changes in white matter microstructure and perceptual speed in individuals aged 80 years and older. Neurobiol Aging. 2016;41:173-86.

21. Bohannon RW. Population representative gait speed and its determinants. J Geriatr Phys Ther. 2008;31(2):49-52.

22. Vetrano DL, Rizzuto D, Calderón-Larrañaga A, Onder G, Welmer A-K, et al. Walking Speed Drives the Prognosis of Older Adults with Cardiovascular and Neuropsychiatric Multimorbidity. Am J Med. 2019;132(10):1207-15. e6.

23. Cesari M, Kritchevsky SB, Penninx BW, Nicklas BJ, Simonsick EM, et al. Prognostic value of usual gait speed in well-functioning older people-results from the health, aging and body composition study. J Am Geriatr Soc. 2005;53(10):1675-80.

24. Dohrn IM, Gardiner PA, Winkler E, Welmer AK. Device-measured sedentary behavior and physical activity in older adults differ by demographic and health-related factors. Eur Rev Aging Phys Act. 2020;17:8.

25. Nelson ME, Rejeski WJ, Blair SN, Duncan PW, Judge JO, et al. Physical activity and public health in older adults: recommendation from the American College of Sports Medicine and the American Heart Association. Med Sci Sports Exerc. 2007;39(8):1435-45.

26. World Health Organization. Global recommendations on physical activity for health. Geneva: World Health Organization; 2010.

27. Rydwik E, Welmer A-K, Kåreholt I, Angleman S, Fratiglioni L, et al. Adherence to physical exercise recommendations in people over 65--the SNAC-Kungsholmen study. Eur J Pub Health. 2013;23(5):799-804.

28. Breslow RA, Chen CM, Graubard BI, Jacobovits T, Kant AK. Diets of drinkers on drinking and nondrinking days: NHANES 2003-2008. Am J Clin Nutr. 2013;97(5):1068-75.

29. Liang Y, Fratiglioni L, Wang R, Santoni G, Welmer AK, et al. Effects of biological age on the associations of blood pressure with cardiovascular and non-cardiovascular mortality in old age: a population-based study. Int J Cardiol. 2016;220:508-13.

30. Marseglia A, Fratiglioni L, Laukka EJ, Santoni G, Pedersen NL, et al. Early cognitive deficits in type 2 diabetes: a population-based study. J Alzheimers Dis. 2016;53(3):1069-78.

31. Buckley DI, Fu R, Freeman M, Rogers K, Helfand M. C-reactive protein as a risk factor for coronary heart disease: a systematic review and metaanalyses for the U.S. preventive services task force. Ann Intern Med. 2009;151(7):483-95.

32. Folstein MF, Folstein SE, McHugh PR. "Mini-mental state". A practical method for grading the cognitive state of patients for the clinician. $J$ Psychiatr Res. 1975;12(3):189-98.

33. Calderon-Larranaga A, Vetrano DL, Onder G, Gimeno-Feliu LA, CoscollarSantaliestra C, et al. Assessing and measuring chronic multimorbidity in the older population: a proposal for its operationalization. J Gerontol A Biol Sci Med Sci. 2017;72(10):1417-23.

34. de Laat KF, van Norden AG, Gons RA, van Oudheusden LJ, van Uden IW, et al. Gait in elderly with cerebral small vessel disease. Stroke. 2010:41(8):1652-8.

35. Su N, Zhai FF, Zhou LX, Ni J, Yao M, et al. Cerebral small vessel disease burden is associated with motor performance of lower and upper extremities in community-dwelling populations. Front Aging Neurosci. 2017;9:313.

36. Ding J, Sigurethsson S, Jonsson PV, Eiriksdottir G, Charidimou A, et al. Large perivascular spaces visible on magnetic resonance imaging, cerebral small vessel disease progression, and risk of dementia: the age, Gene/Environment Susceptibility-Reykjavik Study. JAMA Neurol. 2017:74(9):1105-12.
37. Zheng JJ, Delbaere K, Close JC, Sachdev P, Wen W, et al. White matter hyperintensities are an independent predictor of physical decline in community-dwelling older people. Gerontology. 2012;58(5):398-406.

38. Soumare A, Elbaz A, Zhu Y, Maillard P, Crivello F, et al. White matter lesions volume and motor performances in the elderly. Ann Neurol. 2009;65(6):706-15.

39. Sorond FA, Kiely DK, Galica A, Moscufo N, Serrador JM, et al. Neurovascular coupling is impaired in slow walkers: the MOBILIZE Boston study. Ann Neurol. 2011;70(2):213-20.

40. Ngandu T, Lehtisalo J, Solomon A, Levälahti E, Ahtiluoto S, et al. A 2 year multidomain intervention of diet, exercise, cognitive training, and vascular risk monitoring versus control to prevent cognitive decline in at-risk elderly people (FINGER): a randomised controlled trial. Lancet. 2015;385(9984):2255-63.

41. Richard E, Gouw AA, Scheltens P, Wav G. Vascular Care in Patients with Alzheimer Disease with Cerebrovascular Lesions Slows Progression of white matter lesions on MRI. Stroke. 2010;41(3):554-6.

\section{Publisher's Note}

Springer Nature remains neutral with regard to jurisdictional claims in published maps and institutional affiliations.
Ready to submit your research? Choose BMC and benefit from:

- fast, convenient online submission

- thorough peer review by experienced researchers in your field

- rapid publication on acceptance

- support for research data, including large and complex data types

- gold Open Access which fosters wider collaboration and increased citations

- maximum visibility for your research: over $100 \mathrm{M}$ website views per year

At BMC, research is always in progress.

Learn more biomedcentral.com/submissions 